\title{
A MODIFIED FORM OF THE CENTRAL DIFFERENCE PREDICTOR SCHEME FOR DAMPED NONLINEAR SYSTEMS
}

\author{
S. R. MaruR $\dagger$ and T. KanT $\ddagger$ \\ †CSS Foundation for Research in Finite Elements, B4/4, Vaigai Nagar, Madurai-625 010, India \\ ‡Department of Civil Engineering, Indian Institute of Technology, Powai, Bombay-400 076, India
}

(Received 11 December 1992)

\begin{abstract}
The well-known central difference predictor method used for the analysis of nonlinear systems, subjected to transient dynamic loads, is modified by incorporating Rayleigh's damping into the governing incremental equation of motion. The new time-stepping scheme requires a starting algorithm for both the first and second time-steps, and can effectively be used to analyse the nonlinear damped systems in general.
\end{abstract}

\section{INTRODUCTION}

When any structure is subjected to earthquake, blast, impact or shock loadings, the constituent material becomes plastic and the resulting structural behaviour will be highly nonlinear. In order to predict the nonlinear material behaviour, under transient loadings, the state of the material has to be known between one time-step and the next. As the material behaviour is likely to change during every time-step, the stresses and the internal forces need to be evaluated in such a way that they are based on the exact state of material at any particular time interval.

Moreover damping would be necessary for examining problems where the rate of loading or unloading is very high. Such a condition exists during impact loading and shock propagation. In order to analyse the nonlinear systems under such loadings, the equation of motion with damping in the incremental form, therefore becomes an essential requirement. But it can be seen that many of the works on nonlinear transient dynamic behaviour of structures, considering the equation of motion, either in incremental form [1-8] or in nonincremental form $[9,10]$, do not include damping and the undamped equation of motion is solved by direct integration schemes, to obtain the displacements at every time-step.

Uzgider [12] considered damping in the incremental equation of motion, but using the constant average acceleration method to derive the recursive scheme. The dynamic equilibrium equation with damping has been solved by Shantharam et al. [13] also, using the central difference scheme, but in a nonincremental form.

When the governing equation of motion, available in an incremental form to monitor the state of stress between two successive time-steps, is solved using the central difference scheme due to its established econ- omy in storage and solution efficiency for nonlinear problems $[4,7,15-17]$, the task of probing into the nonlinear behaviour of systems undergoing transient dynamic damped deformations, becomes very much simpler.

To this end, one might consider the well-known central difference predictor scheme [6] in the incremental form, which predicts the incremental acceleration at time $t=t_{n}$ as

$$
\{\Delta \ddot{d}\}_{n}=[M]^{-1}\left[\{\Delta f\}_{n}-\{\Delta p\}_{n}\right],
$$

where $[M]$ is the mass matrix and $\{\Delta f\}_{n}$ and $\{\Delta p\}_{n}$ are the incremental external and internal forces, respectively, at time $t=t_{n}$. The acceleration at the same time is then given as

$$
\{\ddot{d}\}_{n}=\{\ddot{d}\}_{n-1}+\{\Delta \ddot{d}\}_{n} .
$$

Using the central difference operators, the displacements are given as

$$
\{d\}_{n+1}=(\Delta t)^{2}\{\ddot{d}\}_{n}+2\{d\}_{n}-\{d\}_{n-1}
$$

and the incremental displacement vector as

$$
\{\Delta d\}_{n+1}=\{d\}_{n+1}-\{d\}_{n} .
$$

The incremental strains and stresses are evaluated from these displacements. Then the stresses are corrected according to the yield criterion and then using this the internal forces are calculated and are used in eqn (1) for the next time-step.

This available form is very much limited to the undamped systems. As this scheme, when incorporated with the damping parameter, can be used to effectively analyse the damped nonlinear systems, an attempt for a modified form, is made in this study. 


\section{MODIFIED CENTRAL DIFFERENCE PREDICTOR SCHEME}

The equation of dynamic equilibrium for any node $i$ of a structure and any degree of freedom, say rotation $\theta$, can be written at $t=t_{n}$ and $t=t_{n-1}$ as

$$
\left(m_{\theta i}\right)_{n}\left(\ddot{d}_{\theta i}\right)_{n}+\left(c_{\theta i}\right)_{n}\left(\dot{d}_{\theta i}\right)_{n}+\left(p_{\theta i}\right)_{n}=\left(f_{\theta i}\right)_{n}
$$

and

$$
\begin{aligned}
\left(m_{\theta i}\right)_{n-1}\left(\ddot{d}_{\theta i}\right)_{n-1}+\left(c_{\theta i}\right)_{n-1} & \left(\dot{d}_{\theta i}\right)_{n-1} \\
& +\left(p_{\theta i}\right)_{n-1}=\left(f_{\theta i}\right)_{n-1}
\end{aligned}
$$

The equilibrium equation at $t=t_{n}$ can be expressed in incremental form using eqns (5) and (6) as

$$
\left(m_{\theta i}\right)_{n}\left(\Delta \ddot{d}_{\theta i}\right)_{n}+\left(c_{\theta i}\right)_{n}\left(\Delta \dot{d}_{\theta i}\right)_{n}+\left(\Delta p_{\theta i}\right)_{n}=\left(\Delta f_{\theta i}\right)_{n},
$$

where

$$
\begin{aligned}
& \left(\Delta \ddot{d}_{\theta i}\right)_{n}=\left(\ddot{d}_{\theta i}\right)_{n}-\left(\ddot{d}_{\theta i}\right)_{n-1} \\
& \left(\Delta \dot{d}_{\theta i}\right)_{n}=\left(\dot{d}_{\theta i}\right)_{n}-\left(\dot{d}_{\theta i}\right)_{n-1} \\
& \left(\Delta p_{\theta i}\right)_{n}=\left(p_{\theta i}\right)_{n}-\left(p_{\theta i}\right)_{n-1}
\end{aligned}
$$

and

$$
\left(\Delta f_{\theta i}\right)_{n}=\left(f_{\theta i}\right)_{n}-\left(f_{\theta i}\right)_{n-1}
$$

Using the central difference scheme, the acceleration can be given as

$$
\ddot{d}_{n}=\left(d_{n+1}-2 d_{n}+d_{n-1}\right) /(\Delta t)^{2}
$$

and using the eqns (7b) and (8) the incremental acceleration at $t=t_{n}$ can be given as

$$
\begin{aligned}
\left(\Delta \ddot{d}_{\theta i}\right)_{n}=\left[\left(d_{\theta i}\right)_{n+1}-3\left(d_{\theta i}\right)_{n}+\right. & 3\left(d_{\theta i}\right)_{n-1} \\
& \left.-\left(d_{\theta t}\right)_{n}{ }_{2}\right] /(\Delta t)^{2}
\end{aligned}
$$

Similarly the velocity can be expressed in terms of displacements as

$$
d_{n}=\left(d_{n+1}-d_{n-1}\right) / 2(\Delta t)
$$

and the incremental velocity at $t=t_{n}$ can be given using eqns (7c) and (10) as

$$
\begin{aligned}
\left(\Delta d_{\theta i}\right)_{n}=\left[\left(d_{\theta i}\right)_{n+1}\right. & -\left(d_{\theta i}\right)_{n} \\
& \left.-\left(d_{\theta i}\right)_{n-1}+\left(d_{\theta i}\right)_{n-2}\right] / 2(\Delta t)
\end{aligned}
$$

Rayleigh's damping assumes the damping constant to be proportional to the mass [14] and can be expressed as

$$
\left(c_{f i}\right)=\alpha\left(m_{f i}\right)
$$

where $\alpha$ is a constant of proportionality and the incremental equation of motion as given by eqn (7a) can be expressed with the application of eqns (9), (11) and (12) as

$$
\begin{aligned}
& \left(m_{\theta i}\right)\left\{\left(d_{\theta i}\right)_{n+1}-3\left(d_{\theta i}\right)_{n}+3\left(d_{\theta i}\right)_{n-1}-\left(d_{\theta i}\right)_{n-2}\right] /(\Delta t)^{2} \\
& \left.+\alpha\left[\left(d_{\theta i}\right)_{n+1}-\left(d_{\theta i}\right)_{n}-\left(d_{\theta i}\right)_{n-1}+\left(d_{\theta i}\right)_{n-2}\right] / 2(\Delta t)\right\} \\
& +\left(\Delta p_{\theta i}\right)_{n}=\left(\Delta f_{\theta i}\right)_{n}
\end{aligned}
$$

and on rearranging terms yields displacements at $t=t_{n+1}$ as

$$
\begin{aligned}
\left(d_{\theta i}\right)_{n+1}= & \left\{\left[(\Delta t)^{2}\left[\left(\Delta f_{\theta i}\right)_{n}-\left(\Delta p_{\theta i}\right)_{n}\right] /\left(m_{\theta i}\right)\right]\right. \\
& +[3+0.5 \Delta t \alpha]\left(d_{\theta i}\right)_{n}-[3-0.5 \Delta t \alpha]\left(d_{\theta i}\right)_{n-1} \\
& \left.+[1-0.5 \Delta t \alpha]\left(d_{\theta i}\right)_{n-2}\right\} /[1+0.5 \Delta t \alpha]
\end{aligned}
$$

and the displacements along all degrees of freedom of every node, of any discretized continuum, can be evaluated at every time-step, in a similar manner.

\section{STARTING ALGORITHM}

The equation for displacement as given by (14), involves at any time-step $t=t_{n+1}$, information at three previous time steps: $t_{n}, t_{n-1}$ and $t_{n-2}$. Hence special starting algorithms are required for both the first and second time-steps.

\subsection{Starting algorithm for first time-step}

Using the initial displacement and velocity at $t=t_{0}$, the equation of displacement for the first time-step can be arrived at [14]. Acceleration and velocity at $t_{0}$ can be given as

$$
\left(\ddot{d}_{\theta i}\right)_{0}=\left[\left(d_{\theta i}\right)_{1}-2\left(d_{\theta i}\right)_{0}+\left(d_{\theta i}\right)_{-1}\right] /(\Delta t)^{2}
$$

and

$$
\left(\dot{d}_{\theta i}\right)_{0}=\left[\left(d_{\theta i}\right)_{1}-\left(d_{\theta i}\right)_{-i}\right] / 2(\Delta t)
$$

Equation (16) can be rearranged as

$$
\left(d_{i i}\right)_{-1}=\left(d_{t i}\right)_{1}-2(\Delta t)\left(d_{t i}\right)_{0}
$$

With the substitution of eqns (12), (15) and (16), eqn (5) becomes

$$
\begin{aligned}
& \left(m_{\theta i}\right)\left\{\left[\left(d_{\theta i}\right)_{1}-2\left(d_{\theta i}\right)_{0}+\left(d_{\theta i}\right)_{-1}\right] /(\Delta t)^{2}\right. \\
& \left.\left.\quad+\alpha\left[\left(d_{\theta i}\right)_{1}-\left(d_{\theta i}\right)_{-1}\right] / 2(\Delta t)\right\}+\left(p_{\theta i}\right)_{0}=f_{\theta i}\right)_{0}
\end{aligned}
$$

and by applying eqn (17) and rearranging terms yields the displacement for the first time-step as 


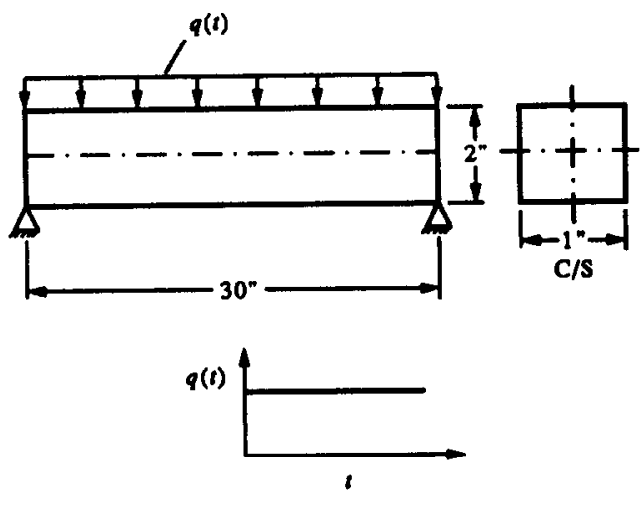

Step pressure

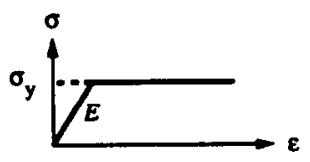

Perfectly plastic material

$$
\begin{aligned}
& E=3 \times 10^{7} \mathrm{psi} \\
& \sigma_{y}=50,000 \mathrm{psi} \\
& q(t)=0.625 \mathrm{po}
\end{aligned}
$$

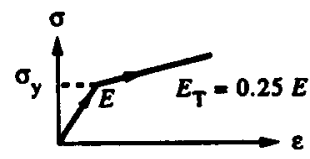

Strain hardening materisl

$\gamma=0.3$

$p=0.733 \times 10^{-3} 1 \mathrm{~b} \sec ^{2} / \mathrm{in}^{4}$

$P_{0}=$ static collapse load
Fig. 1. A simply supported beam with a uniformly distributed transient dynamic load.

$$
\begin{array}{r}
\left(d_{\theta i}\right)_{1}=0.5(\Delta t)^{2}\left\{\left(f_{\theta i}\right)_{0}-\left(p_{\theta i}\right)_{0}\right\} /\left(m_{\theta i}\right)+\left(d_{\theta i}\right)_{0} \\
+(\Delta t)[1-0.5 \Delta t \alpha]\left(d_{\theta i}\right)_{0}
\end{array}
$$

\subsection{Starting algorithm for second time step}

Equation (14) at time $t=t_{2}$ can be expressed as

$$
\begin{aligned}
\left(d_{\theta i}\right)_{2}= & \left\{\left[(\Delta t)^{2}\left[\left(\Delta f_{\theta i}\right)_{1}-\left(\Delta p_{\theta i}\right)_{1}\right] /\left(m_{\theta i}\right)\right]\right. \\
& +[3+0.5 \Delta t \alpha]\left(d_{\theta i}\right)_{1}-[3-0.5 \Delta t \alpha]\left(d_{\theta i}\right)_{0} \\
& \left.+[1-0.5 \Delta t \alpha]\left(d_{\theta i}\right)_{-1}\right\} /[1+0.5 \Delta t \alpha]
\end{aligned}
$$

and after applying eqn (17) and rearranging terms, yields the displacement at the second time-step as

$$
\begin{aligned}
\left(d_{\theta i}\right)_{2}= & \left\{\left[(\Delta t)^{2}\left[\left(\Delta f_{\theta i}\right)_{1}-\left(\Delta p_{\theta i}\right)_{1}\right] /\left(m_{\theta i}\right)\right]\right. \\
& +4\left(d_{\theta i}\right)_{1}-[3-0.5 \Delta t \alpha]\left(d_{\theta i}\right)_{0}-2(\Delta t) \\
& \left.\times[1-0.5 \Delta t \alpha]\left(d_{\theta i}\right)_{0}\right\} /[1+0.5 \Delta t \alpha]
\end{aligned}
$$

\section{FLOW PATTERN OF THE SOLUTION SCHEME}

The displacements at $t=t_{1}$ can be evaluated using eqn (19) and the incremental displacements at the same time-step can be expressed as

$$
\{\Delta d\}_{1}=\{d\}_{1}-\{d\}_{0}
$$

The incremental internal force vector $\{\Delta p\}_{1}$ is evaluated using $\{\Delta d\}_{1}$. With the known $\{\Delta f\}_{1}$, the displacement vector at the next time-step $\{d\}_{2}$, is evaluated using eqn (21). With the quantities like $\{\Delta d\}_{2},\{\Delta p\}_{2}$ and $\{\Delta f\}_{2}$, displacements at $t=t_{3}$ can be calculated from eqn (14). Thereafter the calculation of displacements, internal forces from the incremental displacements and the external forces become cyclic.

\section{NUMERICAL EXPERIMENT}

In order to substantiate the validity of the proposed solution scheme, through numerical experiments, a beam is solved on an IBM compatible, 386 graphic work-station on DOS platform using double precision.

\subsection{Example}

A simply supported beam analysed by Liu and Lin [18], is considered here. As shown in Fig. 1, the beam is subjected to a dynamic step pressure of $0.625 p_{0}$, where $p_{0}$ is the static collapse load of the beam. The beam has been discretized using 12 numbers of two-noded linear elements, with a yield stress of 50,000 psi.

Two types of materials are considered here. The first one is the perfectly-plastic material with the Young's modulus of $3 \times 10^{7} \mathrm{psi}$ and the second one is the strain-hardening material with tangential modulus equal to one fourth of the Young's modulus.

The damping coefficient, considered here can be expressed as [14]

$$
\alpha=2 \xi \omega,
$$

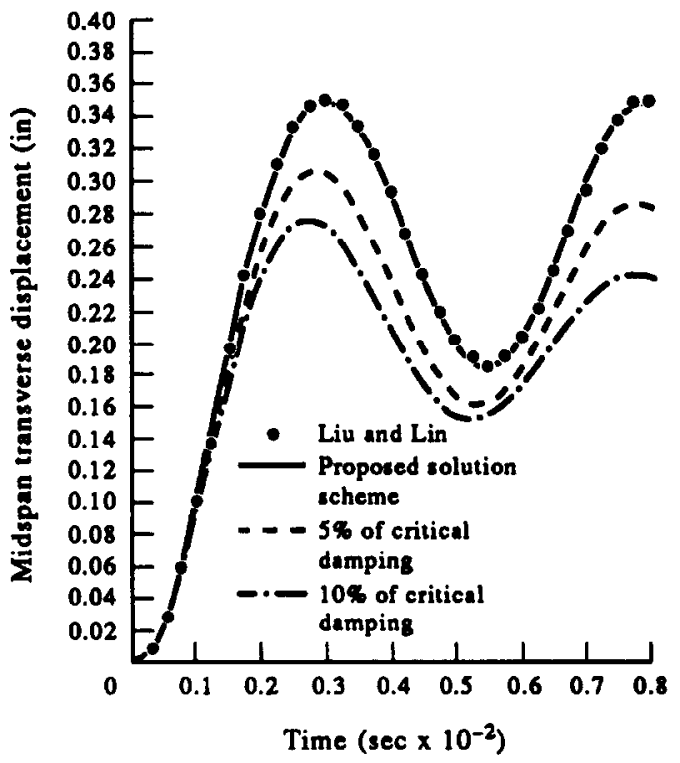

Fig. 2. Damped response history of beam, with a perfectlyplastic material model. 


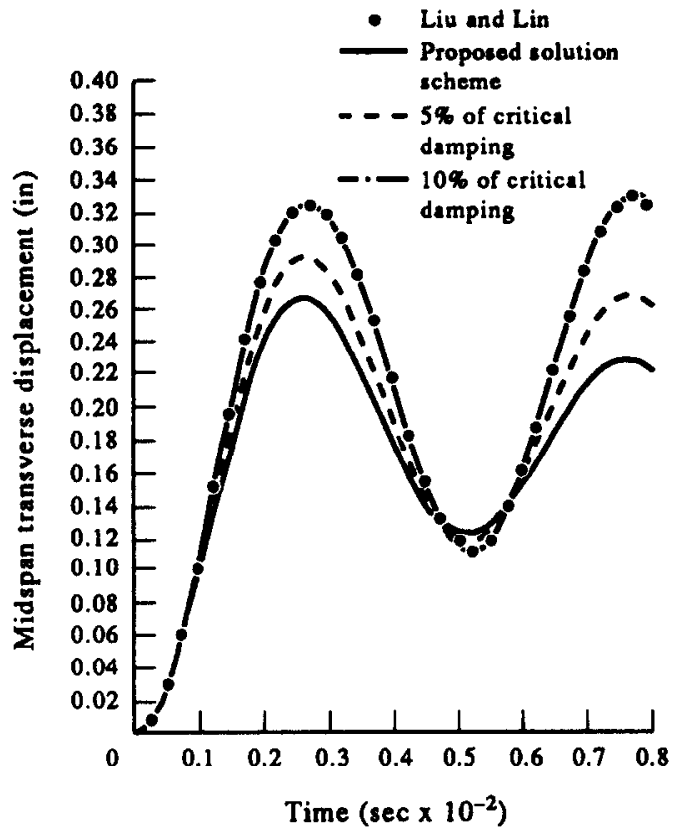

Fig. 3. Damped response history of beam, with a strainhardening material model.

where $\xi$ is the modal damping ratio and $\omega$ is the fundamental frequency. The numerical experiments are conducted for both 5 and $10 \%$ of the critical damping.

The damped and undamped responses of the beam, modelled using the perfectly-plastic and strain-hardening materials are depicted in Figs 2 and 3, respectively.

Through these plots the inherent capability of the proposed modified scheme to predict response of the beam, undergoing damped nonlinear transient dynamic deformations is highlighted. Though an example with simpler boundary conditions and loading mechanisms is chosen for the numerical study, it can be observed that the proposed scheme can handle any complex system, wherein the damping forces manifest predominantly along with the inertial and inelastic forces.

\section{CONCLUSIONS}

A new incremental form of the central difference predictor scheme which incorporates damping is proposed here. This new form requires a starting algorithm for both the first and second time-steps and can be used for evaluating the damped nonlinear dynamic response of structures in a straightforward manner.

\section{REFERENCES}

1. R. W. H. Wu and E. A. Witmer, Finite element analysis of large elastic plastic transient deformations of simple structures. AlAA Jnl 9, 1719-1724 (1971).

2. J. H. Heifitz and C. J. Costantino, Dynamic response of nonlinear media at large strains. ASCE, J. Engng Mech. 98, 1511-1527 (1972).

3. J. L. Bockholt and W. Weaver, Jr, Inelastic dynamic analysis of tier buildings. Comput. Struct. 4, 627-645 (1974).

4. J. F. McNamara, Solution schemes for problems of nonlinear structural dynamics. Trans ASME, J. Press. Vessel Technol 96, 96-102 (1974).

5. S. W. Key, A finite element procedure for large deformation dynamic response of axisymmetric solids. Comput. Meth. appl. Mech. Engng 4, 195-218 (1974).

6. H. Adeli, J. M. Gere and W. Weaver, Jr, Algorithms for nonlinear structural dynamics. ASCE, J. Struct. Div. 104, 263-280 (1978).

7. M. J. Mikkola, M. Tuomala and H. Sinisalo, Comparison of numerical integration methods in the analysis of impulsively loaded elasto plastic and viscoplastic structures. Comput. Struct. 14, 469-478 (1981).

8. T. Y. Kam and S. C. Lin, Nonlinear dynamic analysis of inelastic steel plane frames. Comput. Struct. 28, 535-542 (1988).

9. T. G. Toridis and K. Khozeimeh, Inelastic response of frames to dynamic loads. ASCE, J. Struct. Div. 97, 847-863 (1971).

10. M. Hartzman, Nonlinear dynamic analysis of axisymmetric solids by finite element method. Trans $A S M E, J$. Press. Vessel Technol 96, 103-112 (1974).

11. M. R. Khalil, M. D. Olson and D. L. Anderson, Nonlinear dynamic analysis of stiffened plates. Comput. Struct. 29, 929-941 (1988).

12. E. A. Uzgider, Inelastic response of space frames to dynamic loads. Comput. Struct. 11, 97-112 (1980).

13. D. Shantaram, D. R. J. Owen and O. C. Zienkiewicz, Dynamic transient behaviour of two and three dimensional structures including plasticity, large deformation and fluid interaction. Earthq. Engng Struct. Dyn. 4, 561-578 (1976).

14. D. R. J. Owen and E. Hinton, Finite Elements in Plasticity-Theory and Practice. Pineridge Press, Swansea (1980).

15. H. Adeli, J. M. Gere and W. Weaver, Jr, Algorithms for nonlinear structural dynamics. $A S C E, J$. Struct. Div. 104, 263-280 (1978).

16. R. D. Krieg, Unconditional stability in numerical time integration methods. ASME, J. appl. Mech. 40, 417-421 (1973).

17. J. Mescall and T. Tsui, Influence of damping on the dynamic stability of spherical caps under step pressure loading. AIAA Jnl 9, 1244-1247 (1971).

18. S. C. Liu and T. H. Lin, Elastic-plastic dynamic analysis of structures using known elastic solutions. Eurthy. Engng Struct. Dyn. 7, 147-159 (1979). 